\title{
In situ synthesis of composite nanowires consisting of metal nanoparticles in ion-exchangeable polymer matrix using porous alumina templates
}

\author{
Yurina Fukumoto ${ }^{1)}$, Takaaki Tsuruoka ${ }^{1)}$, Takashi Yanagishita ${ }^{2)}$, Hideki Masuda ${ }^{2)}$, Hidemi \\ Nawafune $^{1)}$ and Kensuke Akamatsu*1) \\ ${ }^{1)}$ Frontiers of Innovative Research in Science and Technology (FIRST), Konan University \\ 7-1-20 Minatojima-minamimachi, Chuo-ku, Kobe 650-0047, Japan \\ e-mail: akamatsu@center.konan-u.ac.jp \\ ${ }^{2)}$ Department of Applied Chemistry, Tokyo Metropolitan Univ. \\ 1-1 Minamiosawa,Hachio-ji,192-0397,Japan
}

\begin{abstract}
One-dimensional (1D) superstructures constructing of inorganic nanoparticles have unique and technologically important potential applications in the areas of nanoelectronics, plasmonics, and molecular sensors. Herein, we describe how nanoporous template and in situ synthetic process can be combined for the construction of composite nanowires consisting of silver nanoparticles dispersed in polymer matrices. We show that, by limiting the space where nanoparticles grow, the silver nanoparticles are encapsulated inward within the polymer nanowires. We also demonstrated that the composite microstructure could be controlled by varying the initial precursor structure, pore size of the template and temperature.

Key words: Composite nanowires, Metal nanoparticles, Porous alumina
\end{abstract}

\section{INTRODUCTION}

Recently, one-dimensional (1D) structures such as wires, rods, belts, tubes and chains have become the focus of intensive reasarch owing to their unique properties [1]. Especially, noble-metal nanoparticle chains have been demonstrated both theoretically and experimentally to be promising candidates for applications in optical and electrical devices [2-4]. The nanoparticle chains can transport optical and electrical energy through the chains below the diffraction limit and has major advantages for scaling down the size of devices. The most important issue for the nanodevices is controllability of $1 \mathrm{D}$ nanostructures such as particle size, separation and shape. Lithographic techniques utilizing atomic force microscopy (AFM) and electron-beam have enabled excellent control of the size and position of metal nanoparticles on the substrate of interest. However, the technique needs substantial time trying to make the materials. One of the low cost approaches is to utilize nanostructured templates such as porous material [5], polymer wires [6-8] and biomolecules [9-11], that previously represents a straightforward route to construct 1D nanostructures. In this approach, the template simply serves as a scaffold within which a different material is generated in situ and shaped into a nanostructure with its morphology complementary to that of the template. Although the existing processes have brought a lot of impressive results, it is still fascinating to develop an new fabrication methods of 1D nanostructures in large quantity and high quality. In this paper, we report a new fabrication method for metal/polymer nanocomposite wires using porous alumina templates, based on in situ-approach using ion-exchangeable polymers [12-15]. We describe fabrication of silver nanoparticle/polymer composite nanowires with diameter and length of few tens of nanometers and several micrometers, respectively. The method relies on introduction of carboxyl groups in polymer and ion exchange reaction, generating metal ion-doped precursors. Subsequently, annealing in hydrogen atmosphere induces reduction of doped metal ions to form metal nanoparticles, and metal/polymer nanocomposite wires are obtained. Furthermore, the size of metal nanoparticles can be controlled by varying concentration of carboxylic acid groups. The process reported herein provide effective methodology for preparing one-dimensional nanocomposite materials.

\section{Experimental section}

Methacrylic acid (MAA), styrene, divinylbenzene were mixed at molar ratio of $5: 3: 2$, respectively, and azobisisobutyronitrile (AIBN) was dissolved into the mixed solution as a polymeric initiator. The solution was then heat-treated at $60{ }^{\circ} \mathrm{C}$ in an $\mathrm{Ar}$ atmosphere for 12 hours after porous alumina template was immersed into the monomer solution. After polymerization of MAA, the template was removed from the polymerized bulk cross-linked PS-PMAA to obtain porous alumina substrates filled with PS-PMAA.

In order to incorporate silver ions into PS-PMAA, the obtained substrates was immersed into a $200 \mathrm{mM}$ aqueous silver nitrite solution at room temperature 


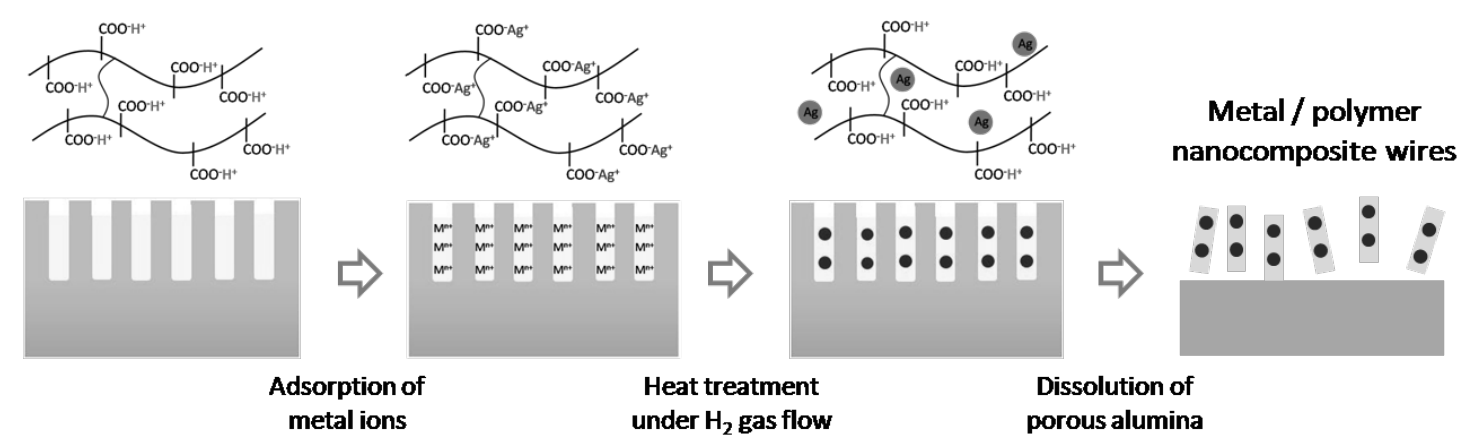

Figure 1. Fabrication process of metal polymer/ nanocomposite wires.

for 36 hours. After rinsing with distilled water, silver ion-doped polymer in porous alumina templates was sealed in a quartz tube under a hydrogen gas flow, followed by annealing in a tube furnace at the rate 10 ${ }^{\circ} \mathrm{C} \min ^{-1}$ up to the target temperature to reduce the silver ions to metal nanoparticles. In order to remove nanowires from the template, the film was dissolved into $500 \mathrm{mM}$ aqueous potassium hydroxide solution for 1 hour. The composite nanowires were obtained by centrifugation using distilled water.

\section{Results and discussion}

In Situ synthesis of silver nanoparticle/polymer nanocomposite- wires

The fabrication scheme of silver/polymer nanocomposite wires is shown in Figure 1. The process consists of 4 steps: filling ion-exchangeable PS-PMAA into nanopores of the alumina templates (step 1), doping of silver ions into polymer nanowires (step 2), reduction of silver ions to form silver nanoparticles (step 3), and removing nanocomposite wires from the templates (step 4). Figure 2a shows the SEM image of porous alumina template, showing regularly arrayed pores (pore diameter: $30 \mathrm{~nm}$ ) [16]. During ion exchange reaction, since metal cations have strong affinity with the carboxylic acid groups, doped silver ions specifically bind to carboxylate anions after ion-exchange reaction, providing silver ion-doped PS-PMAA in nanopores of porous alumina templates.
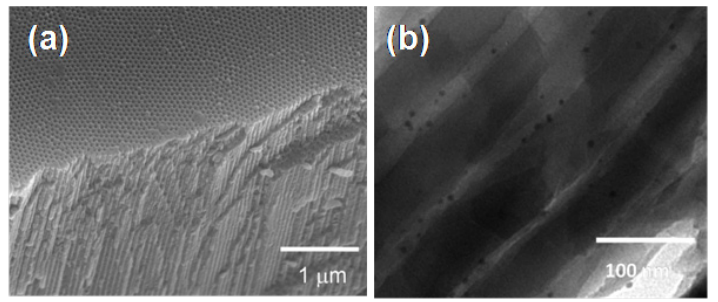

Figure 2. (a) SEM image of porousalumina template. (b) TEM image of sectioned porousalumina with silver nanoparticles dispersed in polymer wires.
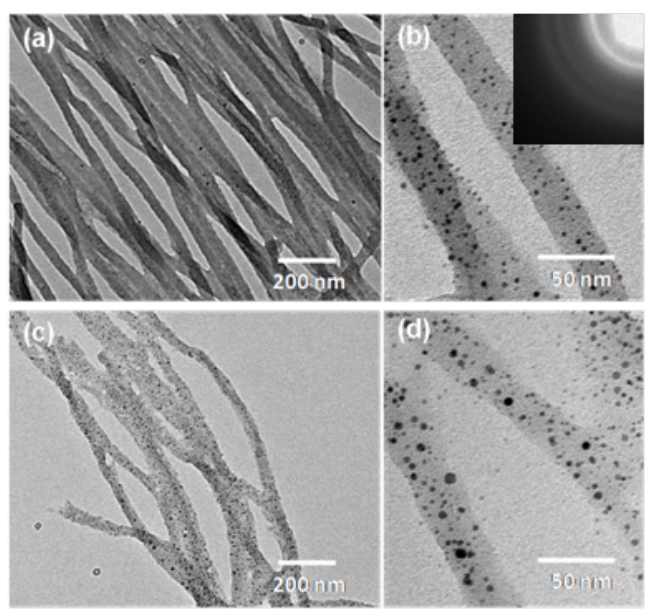

(e)

(f)
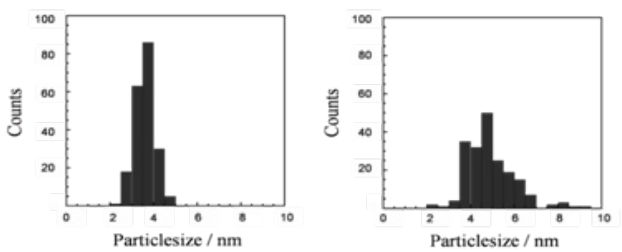

Figure 3. TEM images of silver/polymer nanocomposite wires obtained by carboxylic acid groups content in (a,b) $30 \%$ and $(\mathrm{c}, \mathrm{d}) 70 \%$ PS-PMAA. Size histograms of silver nanoparticles, carboxylic acid groups content in (e) $30 \%$ and (f) $70 \%$ PS-PMAA.

Silver/polymer nanocomposite wires were obtained after heat treatment at $250{ }^{\circ} \mathrm{C}$ for 30 min under $\mathrm{H}_{2}$ gas flow. Figure $2 \mathrm{~b}$ shows the cross-sectional TEM image of composite nanowires in alumina pores before template dissolution. The images show that the silver nanoparticles are grown in nanospace (polymer wires) of the template.

Figure $3 \mathrm{a}$ shows TEM image of the nanowires (initial concentration of carboxylic acid groups: 30 mol\%) obtained after dissolving the templates. The image demonstrates the formation of composite nanowires, and silver nanoparticles were formed homogeneously in the polymer wires. Electron 
diffraction pattern of this sample indicates the formation of metallic silver nanoparticles with fcc crystalline structure (Inset in Fig. 3b). Careful analysis by tilting the sample during TEM observation demonstrated that the silver nanoparticles exist in polymer nanowires (not at nanowire surface). Although silver ions can be introduced only from surface (entrance of pores) of the polymer nanowires during ion exchange reaction, formation of uniform composite nanowires with length of several micrometers indicates that silver ions could diffuse into depth direction of the nanowires (at least up to 5 $\mu \mathrm{m}$ depth after $36 \mathrm{~h}$ ion exchange). Diamater of the composite wires was ca. $28 \mathrm{~nm}$, which is comparable to that of pore diameter of the template (Figure 2a).

Effect of concentration of carboxylic acid groups on silver nanoparticle size

TEM observation was performed for the samples with different contents of carboxylic acid groups in PS-PMAA wires to study the effect of silver ion concentration on particle size. Figure 3(c) shows TEM images of silver nanoparticle/polymer nanowires obtained for samples with initial concentration of carboxylic acid groups of $70 \mathrm{~mol} \%$. As compared to Figure 3a (sample with $30 \mathrm{~mol} \%$ carboxylic acid groups), the size of silver nanoparticles was found to be dependent on the initial concentration of carboxylic acid groups; the greater the content of carboxylic acid groups in the polymer wires, the bigger the silver nanoparticle size. Corresponding size histograms of the dispersed nanoparticles shown in Figure $3 \mathrm{e}$ and $3 \mathrm{f}$ demonstrates that average size of silver nanoparticles are $3.6 \pm 0.4$ $\mathrm{nm}$ and $4.9 \pm 1.1 \mathrm{~nm}$, for samples with $30 \mathrm{~mol} \%$ and $70 \mathrm{~mol} \%$ of carboxylic acid groups, respectively. The results indicate that silver atoms, clusters or smaller nanoparticles were grown to form larger nanoparticles with increasing initial concentration of silver ions. The nanoparticle size could be also controlled by other experimental variables such as heat treatment temperature and heat treatment time: particle size was found to increase with increasing heat treatment temperature and time (results not

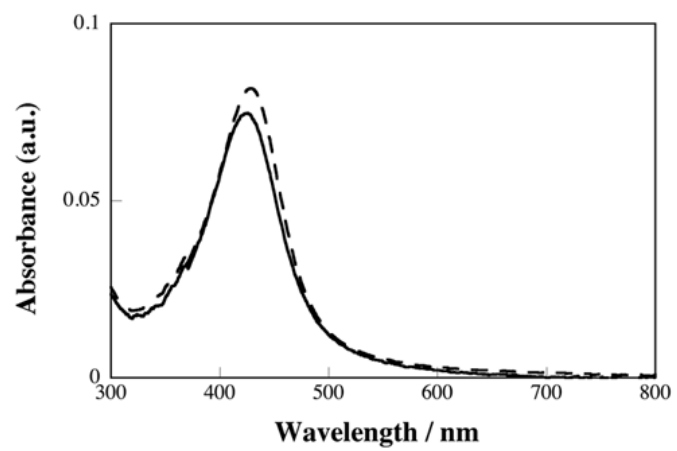

Figure 4. UV-vis spectrum of silver/polymer nanocomposite wires. Initial concentration of carboxylic acid groups: $30 \mathrm{~mol} \%$ (solid line) and $70 \mathrm{~mol} \%$ (dotted line).

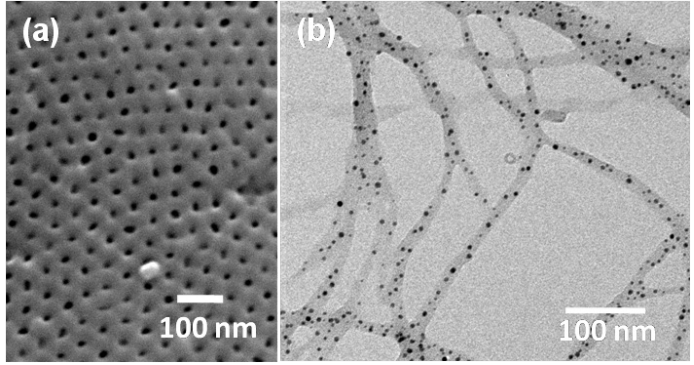

Figure 5. SEM images of porous alumina template and compositewires. Diameter of pore is (a) $15 \mathrm{~nm}$

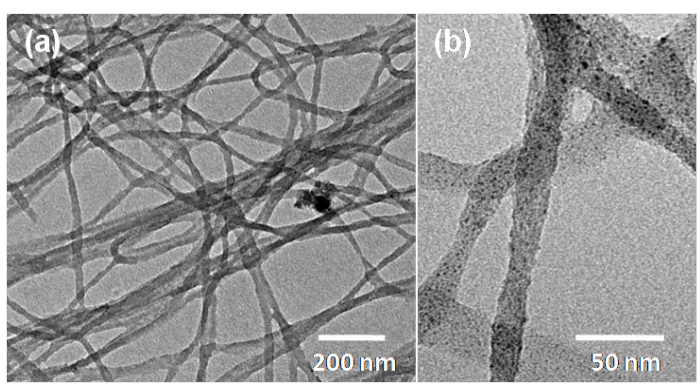

Figure 6. (a) TEM images of Obtained nickel/polymer nanocomposite wires. (b) enlarged image of (a).

shown).

Figure 4 shows UV-vis absorption spectrum of silver/polymer nanocomposite wires dispersed in water. The spectrum shows the absorption peak around $420 \mathrm{~nm}$, which is attributed to surface plasmon resonance (SPR) of silver nanoparticles. The SPR peak position is found to shift toward longer wavelength as the initial concentration of carboxylic acid groups increases, which can be due to increase of nanoparticle size. These results demonstrate that the composite nanowires containing silver nanoparticles uniformly dispersed in polymer matrix can be successfully fabricated through the present in situ approach using porous templates.

\section{Diameter of nanocomposite wires}

The composite nanowires with different diameter could be fabricated using templates with different pore diameter. Figure 5a shows SEM image of the template with pore size of ca. $15 \mathrm{~nm}$. The diameter of nanowires obtained from this template is ca. $16 \mathrm{~nm}$ (Figure 5b), nearly corresponding pore size of the template. In the TEM image, one can see quasi one-dimensional arrangement of silver nanoparticles in the composite nanowires. These results demonstrates that diameter of composite wire is easily controlled by initial pore size of the template.

Nickel/polymer nanocomposite wires

We could also fabricate nickel/polymer nanocomposite wires through present in situ approach. 
In this case, template with polymer nanowires is immersed into $200 \mathrm{mM}$ sodium carbonate solution for $30 \mathrm{~min}$, in order to readily adsorb divalent nickel ions into polymer using $50 \mathrm{mM}$ aqueous nickel chloride solution. Figure 6 shows TEM images of obtained nickel/polymer nanocomposite wires after heat treatment at $250{ }^{\circ} \mathrm{C}$ for $30 \mathrm{~min}$ under $\mathrm{H}_{2}$ gas flow. The image shows that small nickel nanoparticles are dispersed in polymer nanowires. The average size of nickel nanoparticles was ca. $2.5 \mathrm{~nm}$, much smaller than that of silver. This can be caused by lower concentration of divalent nickel ions in nanowires than that of monovalent silver ions.

\section{Conclusion}

In summary, the study has demonstrated a new methodology for preparing composite nanowires consisting of silver nanoparticles dispersed in polymer wires by in situ approach. The microstructure of composite wires, i.e., particle size, diameter of polymer wires can be easily controlled by varying the initial precursor structure (concentration of ion-exchange groups) and pore diameter of porous alumina template, respectively. We also demonstrate the fabrication of nickel/polymer composite nanowires. We believe that this method is simple and versatile for fabrication of various metal/polymer composite nanowires and development of one-dimensional nanodevices.

\section{References}

[1] Y. Xia, P. Yang, Y. Sun, Y. Wu, B. Mayers, B. Gates, Y. Yin, F. Kim, H. Yan, Adv. Mater., 2003, 15, 353

[2] S. A. Maier, P. G. Kik, H. A. Atwater, S. Meltzer, E. Harel, B. E. Koel, A. A. G. Requicha, Nature Mater. 2003, 2, 229

[3] M. Hu, H. Chen, C. Shen, L. Hong, B. Huang, K. Chen, L. Chen, Nature Mater. 2006, 5, 102

[4] J. N. Anker, W. P. Hall, O. Lyandres, N. C. Shah, J. Zhao, R. P. V. Duyne, Nature Mater. 2008, 7, 442

[5] G. Schmid, J. Mater. Chem., 2002, 12, 1231

[6] A. L. Briseno, S. C. B. Mannsfeld, E. Formo, Y. Xiong, X. Lu, Z. Bao, S. A. Jenekhe, Y. Xia, J. Mater. Chem., 2008, 18, 5395

[7] R. Sadar, J. S. Shumaker-Parry, Nano Lett., 2008, 8,731

[8] J. Yuan, F. Schacher, M. Drechsler, A. Hanisch, Y. Lu, M. Ballauff, A. H. E. Müller, Chem. Mater., 2010, 22, 2626

[9] C. Chen, A. L. Rosi, J. Am. Chem. Soc. 2010, 132, 6902

[10] T. Sawada, T. Takahashi, H. Mihara, J. Am. Chem. Soc., 2009, 131, 11434

[11] S. Padalkar, J. R. Capadona, S. J. Rowan, C. Weder, Y. Won, L. A. Stanciu, R. J. Moon, Langmuir, 2010, 26, 8497

[12] S. Ikeda, K. Akamatsu, H. Nawafune, T. Nishio, S. Deki, J. Phys. Chem. B., 2004, 108, 15599

[13] K. Akamatsu, S. Adachi, T. Tsuruoka, S. Ikeda, S. Tomita, H. Nawafune, Chem. Mater., 2008, 20,
3042

[14] K. Akamatsu, H. Shinkai, S. Ikeda, S. Adachi, , H. Nawafune, S. Tomita, J. Am. Chem. Soc., 2005, 127,7980

[15] K. Akamatsu, S. Ikeda, H. Nawafune, S. Deki, Chem. Mater., 2003, 15, 2488

[16] H. Masuda, K. Fukuda, Science, 1995, 268, 1466

(Received January 13, 2012; Accepted February 17, 2012) 